\title{
Factors Determining Birth Intervals: A Multilevel Mixed Effect Parametric Survival Approach
}

\author{
*10. I. Adeniyi, 1I. R. Olonijolu, ${ }^{2}$ A. A. Akinrefon \\ ${ }^{1}$ Department of Statistics, University of Ilorin, llorin, Kwara State, Nigeria \\ ${ }^{2}$ Modibbo Adama University of Technology, Yola, Adamawa State \\ [Corresponding Author: Email: adeniyi.oi@unilorin.edu.ng]
}

\begin{abstract}
Interval between births plays an important role in maternal health as well as child health. This study applies the methodology of Flexible parametric survival models to data on successive births among Nigeria women using the dataset from 2018 National Demographic Health survey. The flexible parametric survival model with Weibull baseline distribution was found to be the best among other fitted baseline distributions. The factors, zone of residence, educational qualification, religion, economic status and age at first birth were found to be significant in predicting the birth intervals. It was found that random effect parameter indicates that the interval between successive births is similar from the same woman.
\end{abstract}

Keywords: Birth intervals, Baseline hazard, Mixed effect, Flexible parametric model, AIC.

\section{INTRODUCTION}

In improving both the maternal and child health, birth spacing plays a crucial role and it is also an essential factor in family planning and fertility control (Adhikari, 2010). Birth interval is the period between successive live births (University of Florida, 2008; John and Kristin, 2019). World Health Organization (WHO) and other international organizations recommend a minimum of 2-3 years interval between pregnancies for the benefit of maternal health and reduction in child and infant mortality. United State Agency for International Development (USAID) suggests that a longer interval of 3-5years might be more beneficial (WHO, 2018).

Both very short and very long inter-birth intervals are associated with health challenges for both the mother and the child (Grundy and Kravdal, 2014). Findings have revealed that short birth intervals, which are less than 24 months are linked to health issues which include maternal morbidities such as uterine rupture and utero-placental bleeding disorders in the women and low birth weight, pre-term birth, small-for-gestational age, stunting growth in the babies (Kozuki et al., 2013; Kozuki and Walker, 2013; Fotso et al., 2013; Adekanmbi et al., 2012; Davanzo et al., 2008; Conde-Agudelo et al., 2006; Conde-Agudelo et al., 2007;
Rustein, 2002). A very long birth interval influences increased risk of pre-eclampsia (Conde-Agudelo, 2012). Moreover, studies found that the risk of pre-eclampsia significantly increased between $10 \%$ to $12 \%$ for each 1 -year increase in inter-pregnancy or birth interval since the first delivery (Skjaerven, et al., 2002). Aside from the health challenges that short birth interval poses, it also accelerates population growth and weakens developmental efforts. It limits the involvement of women in the economic development of their environment and their country at large thereby making them become less productive members of the society (Hailu et al., 2014). The population of Nigeria as at November 2019 was estimated at 203,021,855 based on Worldmeters elaboration of the latest United Nations data which is equivalent to $2.6 \%$ of the total world population and fertility rate of 5.67 (Worldmeters, 2019). Fertility plays an important role in the component of population dynamics as it changes the size and structure of any population (Ayanaw, 2008; Yohannis et al., 2003). Hence, this study aimed to examine birth intervals and its associated factors among women of reproductive age in Nigeria using the parametric multilevel mixed-effect survival time model. 
MATERIALS AND METHODS

Multilevel Mixed Effects Survival Models

In many practical settings, clustered survival data are often observed. A typical application is the case of a recurrent event, where an individual experienced the event of interest in multiple times in the course of the follow-up time (Gutierrez, 2002). In meta-analysis, analysing the individual patient data Individual patient data (IPD) simultaneously within a hierarchical structure allows a direct adjustment for factors and inclusion of non-proportional hazards in covariate effects (Tudur-Smith et al. 2005; Crowther et al., 2012; 2014). Other forms of clustering include the case of individuals living in the same area such as geographical location or patients treated in the same hospital or by the same medical practitioner. Such individuals may share the same unobserved features, such as environmental influence or medical care access (Charvat et al., 2016).

\section{Multilevel Mixed Effect Parametric Survival Models}

Consider the case of a study of G-independent clusters, $i=1, \ldots, G$ (e.g. individuals with repeated events or Hospital, study centres), with each cluster having $j=1, \ldots, n_{i}$ events or individuals. $T_{i j}$ denotes the survival times for individual $j$ from group $i$ and $C_{i j}$ is the corresponding right censoring time. Let $T_{i j}$ be the observed survival time for the $j^{t h}$ individual or event in the $i^{\text {th }}$ cluster or individual, assuming the censoring times are independent of the survival times, the observed times are $Y_{i j}=\min \left(T_{i j}, C_{i j}\right)$ and the censoring indicator $\delta_{i j}=I_{\left\{T_{i j}, C_{i j}\right\}}$ which takes the value of 1 if the event has occurred and 0 if otherwise. For each subject, we observe the explanatory variable, $x_{i j}$.

\section{Application to Data on Birth Interval}

Dataset on repeated five successive birth intervals for 11952 women, aged 15-49 from the 2018 Nigeria Demographic and Health Survey (NDHS) were analysed. Only women who already have five successive births were considered and therefore, there were no censored observations. The birth intervals were recorded in months and the methodology of Flexible parametric survival model was applied. Permission to use data from NDHS (2018) was obtained through online registration with Macro International Incorporation via the DHS website (www.measuredhs.com).

The outcome of interest is the interval between five successive births, each woman considered have experienced five consecutive births with four intervals resulting in a total of 47808 events. Each woman was considered as random because of the repeated birth intervals (events) measured. The geopolitical zone, religion, highest educational qualification, economic status and respondent age at first birth (demographic and economic factors) were considered as explanatory variables. In order to have three categories for the Economic Status variable, the "poorest" and "poorer", from wealth index in NDHS data were combined as "poor", "middle" remained as "middle" while "richer" and "richest" were combined as rich. The two major religions being practiced were considered as Christianity and Islam while the educational qualification was categorised as No education, Primary, Secondary and Higher. The geopolitical zones in the country are NorthCentral, North-East, North-West, South-East, South-South and South-West, respectively while the location of residence is classified as Urban and Rural. The age at first birth is a continuous variable

The overall aim is to fit the Flexible parametric survival model with different baseline parametric distribution. Model assessment was based on Akaike Information Criterion (AIC), (Akaike, 1974) given as:

$A I C=-2 \log L+2 p$

Where $\log L$ is the $\log$ likelihood and $p$ is the number of parameters in the model. A model with lower AIC is preferred.

\section{Ethical Approval}

Permission to use data from NDHS 2018 was obtained through online registration with Macro International Incorporation via the DHS website (www.measuredhs.com). Respondent 
confidentiality was intact as no names and addresses were included in the data set and therefore the respondents cannot be identified by the researcher.

\section{The Proportional Hazards Parametric Survival Model}

The Cox proportional hazards mixed effect survival model is expressed as $h_{i j}(t)=h_{0}(t) \exp \left(\beta^{T} x_{i j}+b_{j}^{T} z_{i j}\right)$

(Yamaguchi, 2002).

where $h_{0}(t)$ is the specified baseline hazard function of any of the distributions; exponential, Weibull or Gompertz, lognormal or log-logistic. $\beta$ is the fixed effect and $b_{j}$ is the random effect, the random effects are assumed to follow a multivariate normal distribution, with $b_{j} \sim N(0, \Sigma)$.

\section{Flexible Parametric Model}

Royston and Parmar (2002) provided the flexible parametric model as an alternative to the traditional proportional hazard. It has the potential for handling repeated event data. It was modelled on the cumulative hazard scale and extended by Crowther et al. (2014) to incorporate random effects. The flexible parametric model is given as;

$H_{i j}(t)=H_{0}(t) \exp \left[x_{i j}^{T} \beta+z_{i j}^{T} b_{j}\right]$

If expressed in log, the model becomes;

$\log \left[H_{i j}(t)\right]=\log \left[H_{0}(t)\right]+X_{i j}^{T} \beta+Z_{i j}^{T} b_{j}$

Where $\beta$ is the fixed effect, $b_{j}$ is the random effect and $H_{0}(t)$ is the cumulative baseline hazard function. The spline basis is derived from the log cumulative hazard function of a Weibull proportional hazards model. The restricted cubic splines were used to relax the linear relationship with log time Royston and Parmar (2002) and Royston and Lambert
(2011). Therefore, the restricted cubic spline function of $\log (t)$, with knots $\boldsymbol{k}_{0}$, as $s\left\{\log (t) \mid \gamma, \boldsymbol{k}_{0}\right\}$. With $\mathrm{K}$ knots if $w=\log (t)$ is written as:

$s\left(w \mid \gamma, \boldsymbol{k}_{0}\right)=\gamma_{0}+\gamma_{1} v_{1}+\gamma_{2} v_{2}+\cdots+$ $\gamma_{m+1} v_{m+1}$ (4).

with derived variables $v_{j}$ (basic functions) and parameter vector $\gamma$, where;

$v_{i}=w$

$v_{j}=\left(w-k_{j}\right)_{+}^{3}-\lambda_{j}\left(w-k_{\min }\right)_{+}^{3}-$

$\left(1-\lambda_{j}\right)\left(w-k_{\max }\right)_{+}^{3}$

for $j=2, \ldots, m+1,\left(w-k_{j}\right)_{+}^{3}$ is equal to $\left(w-k_{j}\right)^{3}$ if the value is positive and 0 elsewhere, and

$\lambda_{j}=\frac{k_{\max }-k_{j}}{k_{\max }-k_{\min }}$

This is now substituted for the log cumulative baseline hazard in equation (3).

$\log \left[H_{i j}(t)\right]=\eta_{i j}=s\left(\log (t) \mid \gamma, k_{0}\right)+$ $X_{i j}^{T} \beta+Z_{i}^{T} b_{i}$

Transforming the hazard and survival scales, we have;

$$
\begin{array}{r}
h_{i j}(t)=\left[\frac{1}{t} \frac{d s\left(\log (t) \mid \gamma, k_{0}\right)}{d \log (t)}\right] \exp \left(\eta_{i j}\right), \\
s_{i j}(t)=\exp \left[-\exp \left(\eta_{i j}\right)\right]
\end{array}
$$

The proportional cumulative hazard is assumed in equation (3). Crowther et al. (2014).

Table 1 presents some probability distributions with their survival, hazard and cumulative survival functions. 
Adeniyi et al. Factors Determining Birth Intervals: A Multilevel Mixed Effect Parametric ...

Table 1: Probability distribution, survival, hazard and cumulative hazard functions of the parametric distributions

\begin{tabular}{|c|c|c|c|c|}
\hline DISTRIBUTIONS & $\begin{array}{c}\text { PROBABILITY } \\
\text { DISTRIBUTION } \\
\text { FUNCTIONS } \\
\end{array}$ & $\begin{array}{c}\text { SURVIVAL } \\
\text { FUNCTIONS }\end{array}$ & $\begin{array}{c}\text { HAZARD } \\
\text { FUNCTIONS }\end{array}$ & $\begin{array}{c}\text { CUMULATIVE } \\
\text { HAZARD } \\
\text { FUNCTIONS }\end{array}$ \\
\hline Exponential & $\lambda e^{-\lambda t}$ & $e^{-\lambda t}$ & $\bar{\lambda}$ & $\lambda t$ \\
\hline Weibull & $\lambda \gamma t^{\gamma-1} e^{-\lambda t^{\gamma}}$ & $e^{-\lambda t^{\gamma}}$ & $\lambda \gamma t^{\gamma-1}$ & $\lambda t^{\gamma}$ \\
\hline Gompertz & $a e^{b t} e^{-\frac{a}{b}\left(e^{b t}-1\right)}$ & $e^{-\frac{a}{b}\left(e^{b t}-1\right)}$ & $a e^{b t}$ & $\frac{a}{h}\left(e^{b t}-1\right)$ \\
\hline Log-logistic & $a b(a t)^{b-1}$ & 1 & $a b(a t)^{b-1}$ & ${ }^{b} \ln (1$ \\
\hline & $\overline{\left(1+(a t)^{b}\right)^{2}}$ & $\overline{1+(a t)^{b}}$ & $\overline{1+(a t)^{b}}$ & $\left.+(a t)^{b}\right)$ \\
\hline Lognormal & $\frac{1}{\sqrt{n}} e^{-\frac{(\ln (t)-m)^{2}}{2 s^{2}}}$ & $(\log (t)-m)$ & $\frac{1}{s t} \Phi\left(\frac{\log (t)-m}{s}\right)$ & \\
\hline & $\sqrt{2} \pi s t$ & $-\Phi\left(\frac{\log (l)-m}{s}\right)$ & $\overline{1-\Phi\left(\frac{\log (t)-m}{s}\right)}$ & \\
\hline \multirow[t]{2}{*}{ Gamma } & $\lambda^{k} t^{k-1} \lambda^{-\lambda t}$ & $1-\mathrm{I}_{k}(\lambda t)$ & $\lambda^{k} t^{k-1} e^{-\lambda t}$ & \\
\hline & $\Gamma(k)$ & & $\left(1-\mathrm{I}_{k}(\lambda k)\right) \Gamma(k)$ & \\
\hline
\end{tabular}

\section{Likelihood and estimation}

Under the mixed effect survival models, the likelihood for the $i^{\text {th }}$ cluster is defined as;

$L_{i}=$

$\int_{-\infty}^{\infty}\left[\prod_{j=1}^{n_{i}} p\left(T_{i j}, \delta_{i j} \mid b_{i}, \boldsymbol{\theta}\right)\right] p\left(b_{i} \mid \boldsymbol{\theta}\right) d b_{i}(10)$

With parameter vector $\theta$, under a hazard model $p\left(T_{i j}, \delta_{i j} \mid b_{i}, \theta\right)=$

$h\left(T_{i j}\right)^{\delta_{i j}} \exp \left[-\int_{0}^{T_{i j}} h\left(T_{i j}\right)\right]$

With $h($ )defined in eq. (1). Assuming proportional hazards under the flexible parametric survival model;

$p\left(T_{i j}, \delta_{i j} \mid b_{i}, \theta\right)=$

$\left[\left\{\frac{1}{T_{i j}} \frac{d s\left(\log \left(T_{i j}\right) \mid \gamma, \mathrm{K}_{0}\right)}{d \log \left(T_{i j}\right)}\right\} \exp \left(\eta_{i j}\right)\right]^{\delta_{i j}} \exp \left\{-\exp \left(\eta_{i j}\right)\right\}$

(12)
The random effects are assumed to follow a multivariate normal distribution

$p\left(b_{i} \mid \theta\right)=(2 \pi|\boldsymbol{V}|)^{-{ }^{q} / 2} \exp \left\{-\frac{b_{i}^{\prime} \boldsymbol{V}^{\prime} b_{i}}{2}\right\}$

(13).

Where $V$ is a variance-covariance matrix and $q$ is the number of random effects. Due to possibly multi-dimensional integral, the integral in equation (10) is analytically intractable and therefore, requires numerical techniques for its evaluation.

\section{RESULTS AND DISCUSSION}

Firstly, the Flexible parametric survival model was fitted with exponential, Weibull, lognormal, loglogistic and gamma baseline distributions. The results showing values of AIC of the models are presented in Table 2.

Table 2: The values of the AIC for Flexible parametric survival models with different baseline distribution

\begin{tabular}{ccc}
\hline BASELINE HAZARD DISTRIBUTION & LOG LIKELIHOOD & AIC \\
\hline Exponential & -214910.5 & 429847.1 \\
Weibull & -34800.38 & 69630.75 \\
Lognormal & -192239.7 & 384509.3 \\
Log logistic & -191798.9 & 383627.8 \\
Gamma & -193777.2 & 387584.3 \\
\hline
\end{tabular}

As observed from Table 2, Flexible parametric survival model with Weibull baseline hazard distribution has the least AIC value and therefore performed best compared to other baseline hazard distributions. Further discussions of effects of the observed factors 
on birth intervals are therefore based on the model with the least AIC value.

The estimates of the hazard ratio and $p$-values for Flexible parametric survival model with Weibull baseline hazard distribution are presented in Table 3. From the p-values, all the factors considered were found to be significant in explaining the variation in the interval of successive births in women. The birth intervals were measured in months, therefore, the hazards increased or decreased by the hazard ratio in months. It was observed that the hazard ratio increased with 0.0271 as the age increased. Findings from EDHS showed similar conclusion (EDHS, 2011). For the different zones of residence, hazard increased with $0.2053,0.3011,0.3191$ and 0.0813 for women from North-east, Northwest, South-east and South-south, respectively compared to women from the North-central but the hazard decreased by 0.1454 for women in the Southwest compared to women from the Northcentral.

Also, for the highest educational qualification, the hazard for women with secondary education increased by 0.0447 compared to women with no education while the hazard decreased for women with primary and higher education with 0.046 and 0.1196 respectively compared to women with no education. The findings on the educational qualification as a determinant of birth interval are consistent with findings of Abdurrahman and Majid (2007), Youssef (2005) and Yohannes et al. (2011). The economic status shows that the hazard of women decreased by 0.0463 and 0.095 for women whose economic status was middle and rich, respectively compared to women whose economic status was poor. The findings on economic status are similar with the result of the study conducted in Ethiopia which revealed that the length of birth interval increased with increasing economic status (Yohannes et al. 2011). The hazard of Christian women decreased by 0.1959 compared to Muslim women.

The estimated frailty standard deviation is 0.5699 (95\% Cl: $0.5545,0.5858)$, indicating a non-heterogeneous baseline hazard function which implies that the interval between repeated births from a woman is similar.

\section{CONCLUSION}

Findings of this study reveal Akaike Information Criterion (AIC) indicate Flexible parametric survival model with Weibull baseline hazard distribution was best to describe the recurrence of birth in individual woman. All the factors considered were found to influence the interval between births. In estimating the random effect parameter, it was revealed that the intervals between successive births are similar from the same woman, which implies that repeated birth intervals from the same woman are homogenous. Therefore, the childbirth spacing is dependent on the individual woman and the intervals between successive births from the same woman are similarly spaced.

Table 3: Hazard ratio, p-value and 95\% confidence interval of the hazard ratio of flexible parametric survival model with Weibull baseline

\begin{tabular}{lcccc}
\hline FACTORS & HAZ. RATIO (S.E) & P-VALUE & \multicolumn{2}{c}{$95 \%$ C.I } \\
\hline Age at first Birth & & & & \\
Age & $1.0271(0.0022)$ & $<0.0001$ & 1.0227 & 1.0314 \\
Zone & & & \\
North-East & $1.2053(0.0295)$ & $<0.0001$ & 1.1488 & 1.2646 \\
North-West & $1.3011(0.0308)$ & $<0.0001$ & 1.2422 & 1.3629 \\
South-East & $1.3191(0.0399)$ & $<0.0001$ & 1.2431 & 1.3997 \\
South-South & $1.0813(0.0348)$ & 0.0150 & 0.8041 & 0.9082 \\
South-West & $0.8546(0.0265)$ & $<0.0001$ & 0.8042 & 0.9082 \\
Highest Educational Qualification & $0.9540(0.0197)$ & 0.0230 & 0.9161 & 0.9935 \\
Primary & & & & \\
\hline
\end{tabular}


Adeniyi et al. Factors Determining Birth Intervals: A Multilevel Mixed Effect Parametric ...

\begin{tabular}{lcccc}
\hline Secondary & $1.0447(0.0256)$ & 0.0750 & 0.9956 & 1.0962 \\
Higher & $0.8804(0.0363)$ & 0.0020 & 0.8121 & 0.9545 \\
Economic Status & & & & \\
Middle & $0.9537(0.0182)$ & 0.0130 & 0.9186 & 0.9901 \\
Rich & $0.9050(0.0185)$ & $<0.0001$ & 0.8694 & 0.9421 \\
Religion & & & & \\
Christian & $0.8041(0.0183)$ & $<0.0001$ & 0.7691 & 0.8407 \\
Constant & $0.0001(0.0000)$ & $<0.0001$ & 0.0001 & 0.0001 \\
Random Effects & & & & \\
Random Effects & & & & \\
Parameters & Estimate & Std. Error & $95 \%$ C.I \\
Woman & 0.5699 & 0.008 & 0.5545 & 0.5858 \\
\hline
\end{tabular}

\section{REFERENCES}

Abdurrahman, R., \& Majid, M. (2007). The determinants of birth interval in AhvazIran: a graphical chain modelling approach. Journal of Data Science, $\mathbf{5}$ : 555-576.

Adekanmbi, V. T, Kayode, G. A., \& Uthman, O. A. (2012). Individual and contextual factors associated with childhood stunting in Nigeria: A multilevel analysis. Maternal and Child Nutrition, 9: 244-259.

Adhikari R. (2010). Demographic, socioeconomic and cultural factors affecting fertility differentials in Nepal. BMC Pregnancy and Child Health, 10:19.

Akaike, $H_{\text {., }}$ A new look at the statistical model identification. IEEE Transactions on Automatic Control, 19(1974): 716-723.

Ayanaw A. (2008). Proximate determinants of birth interval length in Amhara region: the case of Fagita Lekoma district, Awizone. Addis Ababa University College of Development Studies Institute of Population Studies.

Conde-Agudelo, A.; Rosas-Bermudez, A., \& Kafury-Goeta, A.C (2006). Birth spacing and risk of adverse perinatal outcomes: A meta-analysis. The Journal of the American Medical Association, 295:1809-1823.

Conde-Agudelo, A., Rosas-Bermudez, A., \& Kafury-Goeta, A.C. (2007). Effects of birth spacing on maternal health: $A$ systematic review. American Journal of Obstetrics and Gynaecology, 196:297308.
Conde-Agudelo, A., Rosas-Bermudez, A., \& Castano, F. (2012). Effects of birth spacing on maternal, perinatal, infant, and child health: a systematic review of causal mechanisms. Studies in Family. Planning, 43(2): 93-114.

Charvat, H., Remontet, L., Bossard, N., Roche, L., Dejardin, O., Rachet, B., G. Launoy, G., \& Belot, A. (2016). A multilevel excess hazard model to estimate net survival on hierarchical data allowing for non-linear and non-proportional effects of covariates. Statistics in Medicine, 18:3066-84.

Crowther, M. J., R. D. Riley, J. A. Staessen, J. Wang, F. Gueyer, and P. C. Lambert. (2012). Individual patient data metaanalysis of survival data using Poisson regression models. BMC Medical Research Methodology, 12(34):1-14,

Crowther, M. J., Look, M. P., \& Riley, R. D. (2014). Multilevel mixed effects parametric survival models using adaptive Gauss-Hermite quadrature with application to recurrent events and individual participant data metaanalysis. Statistics in Medicine, 33(22): 3844-3858.

Davanzo, J., Hale, L., Razzaque, A., \& Rahman, M. (2008). The effects of pregnancy spacing on infant and child mortality in matlab, Bangladesh: How they vary by the type of pregnancy outcome that began the interval. Population Studies, 62: 131-154.

EDHS. (2011). Central Statistical Agency, Ethiopia Demographic and Health 
Survey 2011: Central Statistical Agency Report, Central Statistical Agency, Addis Ababa, Ethiopia.

Fotso, J.C., Cleland, J., Mberu, B., Mutua, M., \& Elungata, P. (2013). Birth spacing and child mortality: An analysis of prospective data from the Nairobi urban health and demographic surveillance system. Journal of Biosocial Science, 45: 779-798.

Grundy, E., \& Kravdal, Ø. (2014). Do short birth intervals have long-term implications for parental health? Results from analyses of complete cohort Norwegian register data. Journal of Epidemiology Community Health, 68(10): 958-64.

Gutierrez, R. G. (2002). Parametric frailty and shared frailty survival models. The Stata Journal, 2(1), 22-44.

Hailu, D., Gultie, T., \& Workineh, Y. (2014). Barriers to adherence of optimal birth spacing: a qualitative study among mothers and their husbands in Arba Minch Zuria district, Ethiopia. American Journal of Health Research, 2(4):188-95

John, C., Bernstein, S., Ezeh, A., Faundes, A., Glasier, A., \& Innis, J. (2006). Family Planning: the unfinished agenda. The Lancet, 368(9549):1810-1827

John, R. \& Kristin, B. (2019). The Open Birth Interval: A Resource for Reproductive Health Programs and Women's Empowerment. Global Health: Science and Practice, 7(3):355-370.

Kozuki N, Lee AC, Silveira MF, Victora CG, Adair L, Humphrey J, Ntozini R, Black RE, Katz J. (2013). Child Health Epidemiology Reference Group Small-for-GestationalAge-Preterm Birth Working Group. The associations of birth intervals with smallfor-gestational-age, preterm, and neonatal and infant mortality: a meta-analysis. BMC Public Health. 13 Suppl 3(Suppl 3):S3.

Kozuki, N., Walker, N. (2013) Exploring the association between short/long preceding birth intervals and child mortality: using reference birth interval children of the same mother as comparison. BMC Public Health, 13, S6.

Royston, P., \& Parmar, M.K.B. (2002). Flexible Parametric Proportional Hazards and Proportional Odds Models for Censored Survival Data, with Application to Prognostic Modelling and Estimation of
Treatment Effects. Statistics in Medicine, 21(15): 2175-2197.

Royston, P., \& Lambert, P. C. (2011). Flexible Parametric Survival Analysis Using Stata: Beyond the Cox Model. Stata Press.

Rustein, S. (2002). Effects of birth interval on mortality and health: multivariable crosscountry analysis, MACRO International Presentation at USAID, July 2000. In: Setty-Venugoal V, Upadhyay UD: Birth Spacing: Three to Five Saves Lives. Balitmore, Johns Hopkins Bloomberg School of Public Health, Population Information Program, (Population Reports, Series L, Number 13).

Skjaerven, R., Wilcox, A. J., \& Lie, R.T. (2002). The Interval between pregnancies and the risk of pre-eclampsia New England Journal of Medicine, 346, 33-38.

Tudur-Smith, C., Williamson, P. R, Marson, A. G (2005). Investigating heterogeneity in an individual patient data meta-analysis of time to event outcomes. Statistics in Medicine 24(9):1307-19. doi: 10.1002/sim.2050

University of Florida (2008). Repeat Births and average inter-birth intervals among Medicaid Family planning participants, Maternal child health and Education Research and Data centre, College of Florida, Gaineville, Fla, USA.

Worldometers (2019). World population. https://www.worldometers.info/worldpopulation/

Yamaguchi, T., Ohashi, Y., \& Matsuyama, Y. (2002). Proportional hazards models with random effects to examine centre effects in multicentre cancer clinical trials. Statistical Methods for Medical Research, 11(3): 221236.

Yohannes, S., Wondafrash, M., Abera, M., \& Girma, E. (2011). Duration and determinants of birth interval among women of child bearing age in Southern Ethiopia. BMC Pregnancy and Childbirth, 11(38): 1-6.

Yohannis F., Yemane B., \& Alemayehu, W. (2003). Differentials of fertility in rural Butajira. Ethiopian Journal of Health Development, 17(1): 17-25.

Youssef, R. (2005). Duration and determinants of inter-birth interval: community-based survey of women in southern Jordan. The Eastern Mediterranean Health Journal, 11(4): 559-572. 